Research Article

\title{
Fourier-Boas-Like Wavelets and Their Vanishing Moments
}

\author{
Leena Kathuria $\mathbb{D}^{1},{ }^{1}$ Shashank Goel $\mathbb{D},{ }^{1}$ and Nikhil Khanna $\mathbb{D}^{2}$ \\ ${ }^{1}$ Department of Mathematics, Amity Institute of Applied Sciences, Amity University, Sector 125, Noida-201313, U.P., India \\ ${ }^{2}$ Department of Mathematics, Motilal Nehru College, University of Delhi, Delhi-110021, India
}

Correspondence should be addressed to Leena Kathuria; leenakathuria91@gmail.com

Received 10 December 2020; Revised 25 December 2020; Accepted 8 February 2021; Published 8 March 2021

Academic Editor: Efthymios G. Tsionas

Copyright (c) 2021 Leena Kathuria et al. This is an open access article distributed under the Creative Commons Attribution License, which permits unrestricted use, distribution, and reproduction in any medium, provided the original work is properly cited.

\begin{abstract}
In this paper, we propose Fourier-Boas-Like wavelets and obtain sufficient conditions for their higher vanishing moments. A sufficient condition is given to obtain moment formula for such wavelets. Some properties of Fourier-Boas-Like wavelets associated with Riesz projectors are also given. Finally, we formulate a variation diminishing wavelet associated with a Fourier-Boas-Like wavelet.
\end{abstract}

\section{Introduction}

Fourier analysis was initiated with the reconstruction and analysis of periodic functions with the help of Fourier series. It was later extended to Fourier transform in order to study the nonperiodic signals. The Fourier transform is an effective analytical tool to study the continuous and discrete-time signals, but there is a limitation in employing this transform as it does not exhibit the temporal information of the signal. Thus, to overcome this limitation, Gabor [1] introduced the concept of short-time Fourier transform, where an analysis window of fixed length slides over the time axis to give time-localized frequency information. The inherent limitation of short-time Fourier transform lies in the fact that the window width remains the same for all the frequencies, thereby making the localization extent to be constant for different frequencies. The resolution to this problem was given by Grossmann and Morlet [2] who introduced the notion of wavelet transforms in 1984. A function $\psi$ with finite energy, $E_{\psi}$, i.e., $\psi \in L^{2}(\mathbb{R})$, is said to be a wavelet if it satisfies the admissibility condition given by $\mathfrak{夭}_{\psi}=\int_{\mathbb{R}}\left(\left(|\widehat{\psi}(\eta)|^{2}\right) /|\eta|\right) \mathrm{d} \eta<+\infty$, where $\widehat{\psi}$ denotes the Fourier transform of a wavelet $\psi$. The most significant work that has driven the progress of wavelets to great heights was attributed to Mallat [3] and Meyer [4] who both were responsible in the development of the concept of multiresolution analysis (MRA), another way of constructing wavelets.
An MRA consists of a sequence of closed subspaces $\mathscr{V}_{j}, j \in \mathbb{Z}$ of $L^{2}(\mathbb{R})$ satisfying the following conditions: (i) $\mathscr{V}_{j} \subset \mathscr{V}_{j+1}$, for all $j \in \mathbb{Z}$; (ii) $f \in \mathscr{V}_{j}$ if and only if $f(2 \cdot) \in$ $\mathscr{V}_{j+1}$, for all $j \in \mathbb{Z}$; (iii) $\cap_{j \in \mathbb{Z}} \mathscr{V}_{j}=\{0\}$; (iv) $\overline{U_{j \in \mathbb{Z}} \mathscr{V}_{j}}=$ $L^{2}(\mathbb{R}) ;(\mathrm{v})$ if there exists $\phi \in \mathscr{V}_{0}$ so that $\{\phi(\cdot-k): k \in \mathbb{Z}\}$ constitutes an orthonormal basis for $\mathscr{V}_{0}$, then there exists an orthonormal wavelet basis $\psi_{j, k}$ such that $\mathrm{P}_{j} f=\mathrm{P}_{j-1} f+\sum_{k \in \mathbb{Z}}\left\langle f, \psi_{j, k}\right\rangle \psi_{j, k}$ holds, where $\mathrm{P}_{j}$ denotes the orthogonal projection operator onto $\mathscr{V}_{j}$. The function $\phi$ is called a scaling function of the given MRA. It solves the dilation equation $\phi(x)=2 \sum_{k \in \mathbb{Z}} a_{k} \phi(2 x+k)$ with $|\widehat{\phi}(0)|=1$ and $\psi$ is a function associated to $\phi$, which is defined by $\psi(x)=2 \sum_{k \in \mathbb{Z}} b_{k} \phi(2 x+k)$. The functions $\phi$ and $\psi$ are usually known as father and mother wavelets, respectively. It is well established in [5] that a pair of quadrature mirror filter coefficients, $\left(a_{k}\right)_{k \in \mathbb{Z}},\left(b_{k}\right)_{k \in \mathbb{Z}} \in l^{2}(\mathbb{Z})$, is associated to the MRA and the following relations $\widehat{\phi}(\eta)=m_{0}(\eta / 2) \widehat{\phi}(\eta / 2), \widehat{\psi}(\eta)=m_{1}(\eta / 2) \widehat{\phi}(\eta / 2)$ are satisfied for $\eta \in \mathbb{R}$, where $m_{0}$ and $m_{1}$ are given by $m_{0}(\eta)=\sum_{k \in \mathbb{Z}} a_{k} e^{i k \eta}, m_{1}(\eta)=\sum_{k \in \mathbb{Z}} b_{k} e^{i k \eta}=e^{i \eta} \overline{m_{0}(\eta+\pi)}$. For more details on wavelets, one may refer to [6-14].

Boas [15] proposed an integral transform related to the Hilbert transform, which arose due to the study of the class of functions having Fourier transforms vanishing on a finite interval. This transform is known as Boas transform, which finds an application in the theory of filters in electrical 
engineering. Goldberg [16], in 1960, analyzed this transform in detail and gave some significant results and properties. Subsequently, it was further examined by Heywood [17] in 1963 and Zaidi [18] in 1976. For various details pertaining to Boas transform, one may refer to [19].

Khanna et al. [9] introduced Boas transforms of wavelets and furnished various results related to their higher vanishing moments. Later, Khanna and Kathuria [8] studied convolution of these resulting wavelets to analyze Boas transform of convolution of signals. Recently, Khanna et al. [20] introduced fractional Boas transforms and the associated wavelets. These new wavelets appeared to be more prominent than the Boas transforms of wavelets due to an additional degree of freedom in terms of fractional order.

1.1. Framework. This work is streamlined as follows. In Section 2, Fourier-Boas-Like wavelets are introduced and studied. Some results related to higher vanishing moments of such wavelets are obtained. Also, the moment formula for such wavelets by enforcing sufficient condition on the wavelet is derived. Further, some properties of Fourier-Boas-Like wavelets associated with Riesz projectors are given. Finally, a variation diminishing wavelet associated with Fourier-Boas-Like wavelet is constructed.

\section{Fourier-Boas-Like Wavelets}

Let $f \in L^{2}(\mathbb{R})$. Then, the Boas transform of $f$ in terms of principal value integral is defined as

$$
\begin{aligned}
\mathfrak{B} f(x) & =\frac{1}{\pi} p \cdot v \cdot \int_{0}^{\infty} \frac{f(x+z)-f(x-z)}{z^{2}} \sin (z) \mathrm{d} z \\
& =\frac{1}{\pi} p \cdot v \cdot \int_{-\infty}^{\infty} \frac{f(x+z)}{z^{2}} \sin (z) \mathrm{d} z,
\end{aligned}
$$

for any $x$ for which the integral exists.

The relationship between the Boas transform and the Hilbert transform of a function is given by

$$
(\mathfrak{B} f)(x)=(\mathfrak{H} f)(x)-\{\mathfrak{H} f * \mathfrak{g}\}(x),
$$

where

$$
\mathfrak{g}(x)=\left(\frac{2}{\pi}\right)^{(1 / 2)}\left(\frac{1-\cos (x)}{\pi x^{2}}\right),
$$

whereas the Fourier transform and the Hilbert transform share a relationship, specified by

$$
\widehat{\mathfrak{S f}}(\eta)= \begin{cases}-i \widehat{f}(\eta), & \text { if } \eta>0, \\ i \widehat{f}(\eta), & \text { if } \eta<0, \\ 0, & \text { if } \eta=0 .\end{cases}
$$

Taking Fourier transform on both the sides of (2), we have

$$
\widehat{\mathfrak{B} f}(\eta)=\widehat{\mathfrak{H} f}(x)-\mathfrak{F}\{\mathfrak{H} f * \mathfrak{g}\}(\eta)
$$

If $\mathfrak{S} f(x) \in L^{1}(\mathbb{R})$, then using (2), (4), and (5), we obtain $\widehat{\mathfrak{B f}}(\eta)=-i \operatorname{sgn}(\eta) \widehat{f}(\eta)(1-\widehat{\mathfrak{g}}(\eta))$, where

$$
\widehat{\mathfrak{g}}(\eta)= \begin{cases}0, & \text { if }|\eta|>1, \\ 1-|\eta|, & \text { if }|\eta| \leq 1 .\end{cases}
$$

Soares et al. [21] introduced Fourier-like wavelets, defined by $\mathfrak{F}_{\text {Like }}\{\psi\}(x)=(1 / \sqrt{2})(\psi(x)-i \mathfrak{W} \psi(x))$, using the concept of the Fourier kernel $e^{i \eta}$. Further, a factor of $(1 / \sqrt{2})$ was imposed on function $\psi(x)-i \mathfrak{S} \psi(x)$ in order to aver the same energy and admissibility coefficient of its generating wavelet. Later, Khanna et al. [12] defined an improved and natural version of such wavelets by employing Riesz projectors on wavelets. The main idea behind these wavelets was to perlustrate both even and odd symmetries of an asymmetric signal.

One may note that $\mathfrak{B}\{\sin (x)\}=\cos (x)$ and $\mathfrak{B}\{\cos (x)\}=-\sin (x)$, and thus the Fourier kernel can be written as $e^{i x}=\cos (x)-i \mathfrak{B}\{\cos (x)\}$. This observation persuaded us to analyze Boas transform further and we define Fourier-Boas-Like wavelets as $\mathfrak{B}_{\text {Like }}^{\mathfrak{\mho}} \psi(x)=\psi$ $(x)-i \mathfrak{B} \psi(x)$. There are two main reasons for defining these new wavelets: (i) to characterize the wavelets whose Fourier transform vanishes a.e. on ] - 1, 1 [ and (ii) to reinforce the incompetency of wavelets to study both the symmetries of an asymmetric signal.

Next, we give a sufficient condition under which $\mathfrak{B}_{\text {Like }}^{\mathfrak{F}} \psi$ forms a wavelet.

Proposition 1. Let $\psi \in L^{1}(\mathbb{R})$ be a wavelet such that $\widehat{\psi} \in L^{1}(\mathbb{R})$ and $\widehat{\psi}(0)=0$. Then, $\mathfrak{B}_{\text {Like }}^{\mathfrak{F}} \psi$ is again a wavelet.

Proof. Note that

$$
E_{\mathfrak{B}_{\text {Like }}^{\mathfrak{F}} \psi}=\int_{\mathbb{R}}\left|\mathfrak{B}_{\text {Like }}^{\mathfrak{F}} \psi(x)\right|^{2} \mathrm{~d} x \leq \int_{\mathbb{R}}\left(|\psi(x)|^{2}+|\mathfrak{B} \psi(x)|^{2}\right) \mathrm{d} x<+\infty .
$$

Also, we have

$$
\begin{aligned}
\mathfrak{C}_{\mathfrak{B}_{\text {Like }}^{\mathfrak{F}} \psi} & =\int_{\mathbb{R}} \frac{\left|\mathfrak{F}\left\{\mathfrak{B}_{\text {Like }}^{\mathfrak{F}} \psi(x)\right\}(\eta)\right|^{2}}{|\eta|} \mathrm{d} \eta \\
& =\int_{\mathbb{R}} \frac{|\widehat{\psi}(\eta)-\operatorname{sgn}(\eta)(1-\widehat{\mathfrak{g}}(\eta)) \widehat{\psi}(\eta)|^{2}}{|\eta|} \mathrm{d} \eta \\
& <+\infty .
\end{aligned}
$$

Clearly, $\mathfrak{B}_{\text {Like }}^{\mathfrak{F}} \psi$ has finite energy, and it also satisfies the admissibility condition.

Vanishing moments bestow orthogonality relative to subspaces of polynomials and thus perform a significant task in signal processing. For many applications of wavelets such as reconstruction of a signal, compression of images and to examine the regularity of the analyzed signal, a large number of vanishing moments of a wavelet are needed. Theoretically, a large number of vanishing moments insinuate the competency of the scaling function in representing more complex signals scrupulously. A function $f(x)$ is said to 
have $s$ vanishing moments if $\int_{\mathbb{R}} x^{u} f(x) \mathrm{d} x=0,0 \leq u \leq s-1$, where the given integral is called the $u^{\text {th }}$ moment of $f(x)$. For more details, see [10].

Daubechies [22] constructed compactly supported orthonormal wavelets having smoothness of a fixed degree. She observed that if $\phi, \psi \in C^{s}(\mathbb{R})$, then the low-pass filter $m_{0}$ takes the form $m_{0}(\eta)=\left(\left(1+e^{-i \eta}\right) / 2\right)^{s+1} \mathscr{F}(\eta)$, where $\mathscr{F} \in C^{s}(\mathbb{R})$ is $2 \pi$-periodic function. This can be construed using the below stated result which also helps us in deducing that there does not exist any compactly supported orthonormal wavelet $\psi \in C^{\infty}(\mathbb{R})$.

Theorem 1. (see [5]). Let $\psi \in C^{s}(\mathbb{R})\left(s \in \mathbb{N}_{0}\right)$ be a function such that $|\psi(x)| \leq \mathscr{M}(1+|x|)^{-s-1-\varrho}$ for some $\varrho>0$, and that $\psi^{(u)} \in L^{\infty}(\mathbb{R})$ for $u=1,2, \ldots$, s. If $\left\{\psi_{j, k}: j, k \in \mathbb{Z}\right\}$ is an orthonormal system in $L^{2}(\mathbb{R})$, then $\int_{\mathbb{R}} x^{u} \psi(x) d x=0$, for all $u=0,1, \ldots, s$.

Recall from [13] that a function $f$ is said to have fast decay with decay rate $p \in \mathbb{N}$, if there exists a constant $C_{p}$ such that $|f(x)| \leq\left(C_{p} /\left(1+|x|^{p}\right)\right) \forall x \in \mathbb{R}$. The moment formula for the Hilbert transform of $f$ is given by

$$
\mathfrak{S}\left\{x^{s} f(x)\right\}=x^{s} \mathfrak{D} f(x)-\frac{1}{\pi} \sum_{m=0}^{s-1} x^{m} \int_{\mathbb{R}} q^{s-1-m} f(q) \mathrm{d} q, \quad s \geq 0 .
$$

Note that the above formula holds if $x^{s} f(x) \in L^{q}(\mathbb{R}), 1<q<\infty$.

In the following result, we give the relationship between the higher vanishing moments of Fourier-Boas-Like wavelets and the fast decay of wavelet $\psi$.

Theorem 2. Let $\psi, \psi^{(1)}, \widehat{\psi} \in L^{1}(\mathbb{R})$ such that $\psi \in C^{s}(\mathbb{R})$ is having a fast decay with decay exponent $p \in \mathbb{N}$ and $\psi^{(u)} \in L^{\infty}(\mathbb{R}), \quad u=1,2, \ldots, s$. Also, let $x^{s} \psi(x) \in L^{2}(\mathbb{R})$, and $\int_{\mathbb{R}} x^{u} G(x) d x=0$, for $u=0,1, \ldots, s$, where $\quad G(x)=\int_{-1}^{1}(1-(1 /|\eta|)) e^{-2 \pi i \eta x} \widehat{\psi^{(1)}}(-\eta) d \eta$. If $\left\{\psi_{j, k}\right\}_{j, k \in \mathbb{Z}}$ constitutes an orthonormal system in $L^{2}(\mathbb{R})$, then $\int_{\mathbb{R}} x^{u} \mathfrak{B}_{\text {Like }}^{\mathfrak{S}}\{\psi(x)\} d x=0$, for all $u=0,1, \ldots, s$, where $u+1<p$.

Proof. We compute

$$
\begin{aligned}
\int_{\mathbb{R}} x^{u} \mathfrak{B}_{\text {Like }}^{\mathfrak{S}}\{\psi(x)\} \mathrm{d} x & =\int_{\mathbb{R}} x^{u}(\psi(x)-i \mathfrak{B} \psi(x)) \mathrm{d} x \\
& =\int_{\mathbb{R}} x^{u}(\psi(x)-i(\mathfrak{H} \psi(x)-(\mathfrak{H} \psi * \mathfrak{g})(x))) \mathrm{d} x \\
& =\int_{\mathbb{R}} x^{u}\left(\psi(x)-i\left(\mathfrak{S} \psi(x)-\int_{\mathbb{R}} T_{-x} \mathfrak{H} \psi(-t) \mathfrak{g}(t) \mathrm{d} t\right)\right) \mathrm{d} x \\
& =\int_{\mathbb{R}} x^{u}\left(\psi(x)-i\left(\mathfrak{H} \psi(x)-\int_{\mathbb{R}} \mathfrak{F}\left\{\mathfrak{S} T_{-x} \psi\right\}(-\eta) \widehat{\mathfrak{g}}(\eta) \mathrm{d} \eta\right)\right) \mathrm{d} x \\
& =\int_{\mathbb{R}} x^{u}\left(\psi(x)-i\left(\mathfrak{S} \psi(x)+\int_{-1}^{1} \operatorname{sgn}(-\eta) \mathfrak{F}\left\{T_{-x} \psi\right\}(-\eta)(1-|\eta|) \mathrm{d} \eta\right)\right) \mathrm{d} x .
\end{aligned}
$$

Since $\psi, \psi^{(1)}, \widehat{\psi} \in L^{1}(\mathbb{R})$, we have

$$
\begin{aligned}
\int_{\mathbb{R}} x^{u} \mathfrak{B}_{\text {Like }}^{\mathfrak{F}} \psi(x) \mathrm{d} x & =\int_{\mathbb{R}} x^{u}\left(\psi(x)-i\left(\mathfrak{S} \psi(x)-\frac{1}{2 \pi} \int_{-1}^{1}\left(1-\frac{1}{|\eta|}\right) e^{-2 \pi i \eta x} \widehat{\psi^{(1)}}(-\eta) \mathrm{d} \eta\right)\right) \mathrm{d} x \\
& =\int_{\mathbb{R}} x^{u}\left(\psi(x)-i\left(\mathfrak{S} \psi(x)-\frac{1}{2 \pi} G(x)\right)\right) \mathrm{d} x .
\end{aligned}
$$

Now, since $\psi, \widehat{\psi} \in L^{1}(\mathbb{R})$ and $x^{s} \psi(x) \in L^{2}(\mathbb{R})$, it follows that $x^{u} \psi(x) \in L^{2}(\mathbb{R})$, for $u=0,1, \ldots, s$. Using the moment formula for Hilbert transform, (11) can be written as

$$
\int_{\mathbb{R}} x^{u} \mathfrak{B}_{\text {Like }}^{\mathfrak{F}} \psi(x) \mathrm{d} x=\int_{\mathbb{R}}\left(x^{u} \psi(x)-i\left(\mathfrak{H}\left\{x^{u} \psi(x)\right\}+\frac{1}{\pi} \sum_{j=0}^{u-1} x^{j} \int_{\mathbb{R}} q^{u-1-j} \psi(q) \mathrm{d} q\right)\right) \mathrm{d} x .
$$


In view of Theorem 1 and since $x^{u} \psi(x) \in L^{2}(\mathbb{R})$, for $u=0,1, \ldots, s$, it follows that $\int_{\mathbb{R}} x^{u} \mathfrak{B}_{\text {Like }}^{\mathfrak{F}} \psi(x) \mathrm{d} x=0$ for $u=0,1,2, \ldots, s$.

In [14], a sufficient condition was presented in order to obtain higher vanishing moments of wavelets. The result is stated as the following.

Theorem 3. Let $\psi(x)$ be such that for some $s \in \mathbb{N}$, $x^{s} \psi(x), \eta^{s+1} \widehat{\psi}(\eta) \in L^{1}(\mathbb{R})$. If $\left\{\psi_{j, k}(x)\right\}_{j, k \in \mathbb{Z}}$ is an orthogonal system on $\mathbb{R}$, then

$$
\int_{\mathbb{R}} x^{u} \psi(x) \mathrm{d} x=0, \quad \text { for } 0 \leq u \leq s .
$$

Next result generalizes Theorem 3 for Fourier-Boas-Like wavelets.

Theorem 4. Let $\psi, \psi^{(1)}, \widehat{\psi} \in L^{1}(\mathbb{R})$ be such that for some $s \in \mathbb{N}, x^{s} \psi(x) \in L^{1}(\mathbb{R}) \cap L^{2}(\mathbb{R}), \eta^{s+1} \widehat{\psi}(\eta) \in L^{1}(\mathbb{R}), \quad$ and $\int_{\mathbb{R}} x^{u} G(x) d x=0$, for $0 \leq u \leq s$, where $G(x)=\int_{-1}^{1}(1-$ $(1 /|\eta|)) e^{-2 \pi i \eta x} \widehat{\psi^{(1)}}(-\eta) d \eta$. If $\left\{\psi_{j, k}\right\}_{j, k \in \mathbb{Z}}$ is an orthogonal system on $\mathbb{R}$, then $\int_{\mathbb{R}} x^{u} \mathfrak{B}_{\text {Like }}^{\mathfrak{\&}} \psi(x) d x=0$, for $0 \leq u \leq s$.

Proof. We have

$$
\int_{\mathbb{R}} x^{u} \mathfrak{B}_{\text {Like }}^{\mathfrak{F}} \psi(x) \mathrm{d} x=\int_{\mathbb{R}} x^{u} \psi(x) \mathrm{d} x-i \int_{\mathbb{R}} \mathfrak{H}\left\{x^{u} \psi(x)\right\} \mathrm{d} x-\frac{i}{\pi} \sum_{j=0}^{u-1} \int_{\mathbb{R}} x^{j} \int_{\mathbb{R}} q^{u-1-j} \psi(q) \mathrm{d} q \mathrm{~d} x .
$$

Since $x^{u} \psi(x) \in L^{2}(\mathbb{R})$ for $u=0,1, \ldots, s$, by Theorem 3 , it follows that $\int_{\mathbb{R}} x^{u} \mathfrak{B}_{\mathrm{Like}}^{\mathfrak{F}} \psi(x) \mathrm{d} x=0$, for $u=0,1,2, \ldots, s$.

In [6], regularity of orthonormal wavelet bases was studied and a relationship between the regularity of wavelet and the multiplicity of the zero at $\eta=0$ of $\widehat{\psi}$ was observed. This observation can be seen and deduced using the following result which is given in more generalized form.

Theorem 5. Let $f, \tilde{f}$ be two functions (not identically constant) such that

(i) $\left\langle f_{j, k}, \tilde{f}_{j_{l}, k_{l}}\right\rangle=\delta_{j j_{1}} \delta_{k k_{\prime}}, \quad$ where $f_{j, k}(x)=2^{j / 2}$ $f\left(2^{j} x-k\right), \tilde{f}_{j, k}(x)=2^{j / 2} \tilde{f}\left(2^{j} x-k\right)$.

(ii) $|\tilde{f}(x)| \leq M(1+|x|)^{-\gamma}$, with $\gamma>s+1$.

(iii) $f \in C^{s}$, with $f^{(u)}$ bounded for $u \leq s$.

Then, $\int_{\mathbb{R}} x^{u} \tilde{f}(x) d x=0$ for $u=0,1, \ldots, s$.

The next result generalizes Theorem 5 and depicts the relationship among the regularity of orthonormal wavelets $\psi$ and vanishing moments of Fourier-Boas-Like wavelets.

Theorem 6. Let $\psi, \psi^{(1)}, \widehat{\psi} \in L^{1}(\mathbb{R})$ and let the system $\psi_{j, k}=2^{j / 2} \psi\left(2^{j} x-k\right), j, k \in \mathbb{Z}$ form an orthonormal set in $L^{2}(\mathbb{R})$ such that $|\psi(x)| \leq M(1+|x|)^{-s-1-\varrho}$, where $\varrho>0$ and $\psi \in C^{s}(\mathbb{R})$ such that $\psi^{(u)}$ is bounded for $u \leq s$. Also, if $x^{s} \psi(x) \in L^{2}(\mathbb{R})$ and $\int_{\mathbb{R}} x^{u} G(x) d x=0$, for $0 \leq u \leq s$, where $G(x)=\int_{-1}^{1}(1-(1 /|\eta|)) e^{-2 \pi i \eta x} \widehat{\psi^{(1)}}(-\eta) d \eta$, then $\int_{\mathbb{R}} x^{u} \boldsymbol{B}_{\text {Like }}$ $\mathfrak{F}\{\psi(x)\} d x=0$ for $u=0,1,2, \ldots, s$.

Proof. Note that

$$
\begin{aligned}
& \int_{\mathbb{R}} x^{u} \mathfrak{B}_{\text {Like }}^{\mathfrak{Y}}\{\psi(x)\} \mathrm{d} x=\int_{\mathbb{R}} x^{u} \psi(x) \mathrm{d} x-i \int_{\mathbb{R}} \mathfrak{H}\left\{x^{u} \psi(x)\right\} \mathrm{d} x \\
& -\frac{i}{\pi} \sum_{j=0}^{u-1} \int_{\mathbb{R}} x^{j} \int_{\mathbb{R}} q^{u-1-j} \psi(q) \mathrm{d} q \mathrm{~d} x .
\end{aligned}
$$

Therefore, in view of Theorem 5 and the fact that $x^{u} \psi(x) \in L^{2}(\mathbb{R})$, for $u=0,1, \ldots, s, \quad$ it follows that $\int_{\mathbb{R}} x^{u} \mathfrak{B}_{\text {Like }}^{\mathscr{F}} \psi(x) \mathrm{d} x=0$, for $u=0,1,2, \ldots, s$.

Recall from [12] that wavelets associated with projection operators $\mathbb{P}_{+}$and $\mathbb{P}_{-}$involving the Hilbert transform are defined as $\mathbb{P}_{+}=(1 / 2)(\mathfrak{I}+i \mathfrak{S})$ and $\mathbb{P}_{-}=(1 / 2)(\mathfrak{I}-i \mathfrak{H})$, where $\mathfrak{I}$ and $\mathfrak{H}$ represent the identity operator and Hilbert transform operator, respectively. These projection operators are also known as Riesz projectors.

In the following result, the moment formula for Fourier-Boas-Like wavelets is given.

Theorem 7. Let $\psi \in L^{1}(\mathbb{R})$ be a wavelet such that $\widehat{\psi} \in L^{1}(\mathbb{R})$ and $x^{s} \psi(x) \in L^{1}(\mathbb{R}) \cap L^{2}(\mathbb{R})$, for some $s \in \mathbb{N}$. Then,

$$
\begin{aligned}
\mathfrak{B}_{\text {Like }}^{\mathfrak{F}}\left\{x^{s} \psi(x)\right\}= & 2 x^{s} \mathbb{P}_{-} \psi(x)+\frac{i}{\pi} \sum_{p=0}^{s-1} x^{p} \int_{\mathbb{R}} q^{s-1-p} \psi(q) \mathrm{d} q \\
& +\left(\frac{-1}{2 \pi i}\right)^{s} \int_{-1}^{1} \widehat{\psi}^{(s)}(\eta) e^{-2 \pi i \eta x}(\operatorname{sgn}(\eta)-\eta) \mathrm{d} \eta,
\end{aligned}
$$

where $\mathbb{P}_{-} \psi$ is a wavelet associated with Riesz projectors.

Proof. We compute 


$$
\begin{aligned}
\mathfrak{B}_{\text {Like }}^{\mathfrak{F}}\left\{x^{s} \psi(x)\right\}= & x^{s} \psi(x)-i \mathfrak{B}\left\{x^{s} \psi(x)\right\}=2 x^{s} \mathbb{P}_{-} \psi(x)+\frac{i}{\pi} \sum_{p=0}^{s-1} x^{p} \int_{\mathbb{R}} q^{s-1-p} \psi(q) \mathrm{d} q \\
& +i \int_{\mathbb{R}} \mathfrak{F}\left\{\mathfrak{H}\left\{t^{s} \psi(t)\right\}\right\}(\eta) \mathfrak{F}\left\{T_{x} \mathfrak{g}(t)\right\}(\eta) \mathrm{d} \eta=2 x^{s} \mathbb{P}_{-} \psi(x)+\frac{i}{\pi} \sum_{p=0}^{s-1} x^{p} \int_{\mathbb{R}} q^{s-1-p} \psi(q) \mathrm{d} q \\
& +i \int_{\mathbb{R}}-i \operatorname{sgn}(\eta)\left\{\mathfrak{F}\left\{t^{s} \psi(t)\right\}\right\}(\eta) E_{-x} \widehat{\mathfrak{g}}(\eta) \mathrm{d} \eta=2 x^{s} \mathbb{P}_{-} \psi(x)+\frac{i}{\pi} \sum_{p=0}^{s-1} x^{p} \int_{\mathbb{R}} q^{s-1-p} \psi(q) \mathrm{d} q \\
& +\left(\frac{-1}{2 \pi i}\right)^{s} \int_{-1}^{1} \widehat{\psi}^{(s)}(\eta) e^{-2 \pi i \eta x}(\operatorname{sgn}(\eta)-\eta) \mathrm{d} \eta .
\end{aligned}
$$

A continual filter is defined by $\varphi(f(x))=\mathbb{P}_{-} f(x)$, where $f \in L^{2}(\mathbb{R})$ and $\varphi: L^{2}(\mathbb{R}) \longrightarrow L^{2}(\mathbb{R})$. Note that $\varphi$ is said to be a convolution filter if $\varphi(x)=w * x$ for any $x$, where $w \in\left(L^{1} \cap L^{2}\right)(\mathbb{R})$ is a weight function and $\widehat{w}(\eta)$ is known as the transfer function. For more details, one may see [12].

In the next result, we give a sufficient condition under which $\mathbb{P}_{-}\left\{\mathfrak{B}_{\text {Like }}^{\mathfrak{F}} \psi\right\}(x)$, i.e., Fourier-Boas-Like wavelets associated with Riesz projectors form a convolution filter and Fourier transform of $\mathbb{P}_{-}\left\{\mathfrak{B}_{\text {Like }}^{\mathfrak{\mho}} \psi\right\}(x)$ vanishes for all positive frequencies.

Theorem 8. Let $\psi \in L^{1}(\mathbb{R})$ be a wavelet such that $\widehat{\psi} \in L^{1}(\mathbb{R})$ and $\widehat{\psi}(0)=0$. Then, $\mathbb{P}_{-}\left\{\mathfrak{B}_{\text {Like }}^{\mathfrak{F}} \psi\right\}(x)$ is a convolution filter with transfer function

$$
\begin{cases}2, & \text { if } \eta<-1, \\ 1-\eta, & \text { if }-1 \leq \eta<0 \\ \frac{1}{2}, & \text { if } \eta=0 \\ 0, & \text { if } \eta>0,\end{cases}
$$

and Fourier transform of $\mathbb{P}_{-}\left\{\mathfrak{B}_{\text {Like }}^{\mathfrak{F}} \psi\right\}(x)$ vanishes for all positive frequencies.

Proof. Note that

$$
\begin{aligned}
\varphi\left(\mathfrak{B}_{\text {Like }}^{\mathfrak{\&}} \psi(x)\right) & =\mathbb{P}_{-}\left\{\mathfrak{B}_{\text {Like }}^{\mathfrak{F}} \psi\right\}(x) \\
& =\frac{1}{2}\left(\mathfrak{B}_{\text {Like }}^{\mathfrak{F}} \psi(x)-i\left(\frac{1}{\pi} p \cdot v \cdot \frac{1}{x} * \mathfrak{B}_{\text {Like }}^{\mathfrak{F}} \psi(x)\right)\right) \\
& =\left(\mathbb{P}_{-} \delta * \mathfrak{B}_{\text {Like }}^{\mathfrak{\&}} \psi\right)(x) \\
& =\left(\mathbb{P}_{-} \delta * \psi\right)(x)-i\left(\mathbb{P}_{-} \delta *(\mathfrak{S} \psi-\mathfrak{S} \psi * \mathfrak{g})\right)(x) \\
& =\left(\mathbb{P}_{-} \delta * \psi\right)(x)-i \mathfrak{B}\left(\mathbb{P}_{-} \delta * \psi\right)(x) .
\end{aligned}
$$

Thus, $\mathbb{P}_{-}\left\{\mathfrak{B}_{\text {Like }}^{\mathfrak{Y}} \psi\right\}(x)$ is a convolution filter. Clearly, we calculate

$$
\begin{aligned}
\mathfrak{F}\left\{\mathfrak{B}_{\text {Like }}^{\mathfrak{F}}\left\{\mathbb{P}_{-} \delta\right\}\right\}(\eta)= & \mathfrak{F}\left\{\mathbb{P}_{-} \delta\right\}(\eta)[1-\operatorname{sgn}(\eta)(1-\widehat{\mathfrak{g}}(\eta))] \\
& = \begin{cases}2, & \text { if } \eta<-1, \\
1-\eta, & \text { if }-1 \leq \eta<0, \\
\frac{1}{2}, & \text { if } \eta=0, \\
0, & \text { if } \eta>0 .\end{cases}
\end{aligned}
$$

Also, we evaluate

$$
\mathfrak{F}\left\{\mathbb{P}_{-}\left\{\mathfrak{B}_{\text {Like }}^{\mathfrak{\mho}} \psi\right\}\right\}(\eta)= \begin{cases}2 \widehat{\psi}(\eta), & \text { if } \eta<-1, \\ (1-\eta) \widehat{\psi}(\eta), & \text { if }-1 \leq \eta<0, \\ \frac{\widehat{\psi}(\eta)}{2}, & \text { if } \eta=0, \\ 0, & \text { if } \eta>0 .\end{cases}
$$

Let $\alpha(x) \in L^{1}(\mathbb{R})$ and let $h(x)=(\alpha * \psi)(x)=\int_{\mathbb{R}} \alpha$ $(x-t) \psi(t) \mathrm{d} t$, where $\psi(t)$ is continuous and bounded. The kernel $\alpha(x)$ is variation diminishing if $\eta[\alpha * \psi] \leq \eta[\psi]$, where $\eta[\psi]$ denotes number of changes of sign of $\psi$ on $\mathbb{R}$. It was found that $\alpha(x)$ is variation diminishing if and only if

$$
\widehat{\alpha}(\eta)=\int_{\mathbb{R}} \alpha(x) e^{-i \eta x} \mathrm{~d} x=\left(e^{l \eta^{2}+i m \eta} \prod_{n \in \mathbb{N}}\left(1-\frac{i \eta}{d_{n}}\right) e^{i \eta / d_{n}}\right)^{-1},
$$

where $l, m \neq 0$ and $d_{n}(n \in \mathbb{N})$ are real numbers with $\sum_{n \in \mathbb{N}} d_{n}^{-2}$ $<\infty$.

This gives 


$$
\begin{aligned}
& \alpha(x)=\frac{1}{2 \pi} \int_{\mathbb{R}} e^{i \eta x}\left(e^{l \eta^{2}+i m \eta} \prod_{n \in \mathbb{N}}\left(1-\frac{i \eta}{d_{n}}\right) e^{i \eta / d_{n}}\right)^{-1} \mathrm{~d} \eta \\
& \alpha^{(s)}(x)=\frac{1}{2 \pi} \int_{\mathbb{R}}(i \eta)^{s} e^{i \eta x}\left(e^{l \eta^{2}+i m \eta} \prod_{n \in \mathbb{N}}\left(1-\frac{i \eta}{d_{n}}\right) e^{i \eta / d_{n}}\right)^{-1} \mathrm{~d} \eta .
\end{aligned}
$$

Let $\psi$ be a wavelet. Then, for a variation diminishing kernel given by (23) such that $\sum_{n \in \mathbb{N}} d_{n}^{-2}<\infty, \psi * \alpha$ is known as wavelet, a wavelet with specific changes of sign. For further details, one may read [23, 24].

In the given result, we show that $\mathfrak{B}_{\text {Like }}^{\mathfrak{F}} \psi * \alpha$ is also a variation diminishing wavelet.

Theorem 9. Let $\psi \in L^{1}(\mathbb{R})$ be a wavelet such that $\widehat{\psi} \in L^{1}(\mathbb{R})$ and $\widehat{\psi}(0)=0$. Let $\alpha \in L^{1}(\mathbb{R})$ be a variation diminishing kernel. Then, $\mathfrak{B}_{\text {Like }}^{\mathfrak{F}} \psi * \alpha$ is a variation diminishing wavelet.

Proof. Since $\mathfrak{B}_{\text {Like }}^{\mathfrak{\mho}} \psi \in L^{2}(\mathbb{R})$ and $\alpha \in L^{1}(\mathbb{R})$, it follows that $\mathfrak{B}_{\text {Like }}^{\mathfrak{\mho}} \psi * \alpha \in L^{2}(\mathbb{R})$.

Also note that

$$
\begin{aligned}
\left\|\mathfrak{B}_{\text {Like }}^{\mathfrak{S}} \psi\right\|_{1} & =\|\psi-i \mathfrak{B} \psi\|_{1} \\
& =\|\psi-i \mathfrak{S} \psi+i \mathfrak{S} \psi * \mathfrak{g}\|_{1} \\
& \leq\|\psi\|_{1}+\|\mathfrak{S} \psi\|_{1}+\|\mathfrak{S} \psi * \mathfrak{g}\|_{1} .
\end{aligned}
$$

Since $\widehat{\psi} \in L^{1}(\mathbb{R})$ and $\widehat{\psi}(0)=0$, it follows that $\mathfrak{S} \psi \in L^{1}(\mathbb{R})$. Thus,

$$
\left\|\mathfrak{B}_{\text {Like }}^{\mathfrak{F}} \psi\right\|_{1} \leq\|\psi\|_{1}+\|\mathfrak{S} \psi\|_{1}+\|\mathfrak{S} \psi\|_{1}\|\mathfrak{g}\|_{1}<+\infty .
$$

Hence,

$$
\begin{aligned}
\mathfrak{S}_{\mathfrak{B}_{\text {Like }}^{\mathfrak{F}} \psi * \alpha} & =\int_{\mathbb{R}} \frac{\left|\mathfrak{F}\left\{\mathfrak{B}_{\text {Like }}^{\mathfrak{S}} \psi * \alpha\right\}(\eta)\right|^{2}}{|\eta|} \mathrm{d} \eta, \\
& =\int_{\mathbb{R}} \frac{\left|\mathfrak{F}\left\{\mathfrak{B}_{\text {Like }}^{\mathfrak{F}} \psi\right\}(\eta)\right|^{2}|\widehat{\alpha}(\eta)|^{2}}{|\eta|} \mathrm{d} \eta \\
& \leq \begin{cases}4 \mathfrak{D} \mathfrak{S}_{\psi}, & \text { if } \eta<-1, \\
\mathfrak{D} \int_{\mathbb{R}} \frac{|\psi(\eta)(1-\eta)|^{2}}{|\eta|} \mathrm{d} \eta, & \text { if }|\eta| \leq 1, \eta \neq 0, \\
\mathfrak{D} \mathfrak{S}_{\psi}, & \text { if } \eta=0, \\
0, & \text { if } \eta>1,\end{cases} \\
& \leq \begin{cases}4 \mathfrak{A} \mathfrak{S}_{\psi}, & \text { if } \eta<-1, \\
4 \mathfrak{A} \mathfrak{S}_{\psi}, & \text { if }|\eta| \leq 1, \eta \neq 0, \\
\mathfrak{A} \mathfrak{S}_{\psi}, & \text { if } \eta=0, \\
0, & \text { if } \eta>1,\end{cases} \\
& +\infty, \quad
\end{aligned}
$$

where $\mathfrak{D}=\sup _{\eta}\left|e^{i \eta^{2}+i m \eta} \prod_{n \in \mathbb{N}}\left(1-\left(i \eta / d_{n}\right)\right) e^{\left(i \eta / d_{n}\right)}\right|^{-2}$ and $\mathfrak{A}=$ $\sup \left(1 /\left(\prod_{n \in \mathbb{N}}\left(1-\left(\eta^{2} / d_{n}^{2}\right)\right)\right)\right)$.

\section{Conclusions}

New wavelet functions, called Fourier-Boas-Like wavelets, have been established. These wavelets have been found to be better than the earlier proposed wavelets in $[12,21]$ derived from the Riesz projectors and Fourier kernels, respectively. Various results related to higher vanishing moments of Fourier-Boas-Like wavelets have been given, and it is observed that regularity and fast decay are significant attributes for the vanishing moments of Fourier-Boas-Like wavelets. It has also been investigated that under some conditions, Fourier-Boas-Like wavelets associated with Riesz projectors form a convolution filter. Further, using Schoenberg's theory of variation diminishing integral operators of convolution type, variation diminishing wavelet associated with Fourier-Boas-Like wavelet is constructed.

\section{Data Availability}

No data were used to support this study.

\section{Conflicts of Interest}

The authors declare that they have no conflicts of interest.

\section{Authors' Contributions}

All authors contributed equally to this paper and read and approved the final manuscript.

\section{References}

[1] D. Gabor, "Theory of communication. part 1: the analysis of information," Journal of the Institution of Electrical EngineersPart III: Radio and Communication Engineering, vol. 93, no. 26, pp. 429-441, 1946.

[2] A. Grossmann and J. Morlet, "Decomposition of Hardy functions into square integrable wavelets of constant shape," SIAM Journal on Mathematical Analysis, vol. 15, no. 4, pp. 723-736, 1984.

[3] S. G. Mallat, "Multiresolution approximations and wavelet orthonormal bases of $L^{2}(\mathbb{R})$," Transactions of the American Mathematical Society, vol. 315, no. 1, p. 69, 1989.

[4] Y. Meyer, Wavelets and Operators, Cambridge University Press, Cambridge, UK, 1992.

[5] E. Hernández and G. Weiss, A First Course on Wavelets, CRC Press, Boca Raton, FL, USA, 1996.

[6] I. Daubechies, "Ten lectures on wavelets," in CBMS-NSF Regional Conference Series in Applied MathematicsSociety for Industrial and Applied Mathematics (SIAM), Philadelphia, PA, USA, 1992.

[7] L. Debnath and F. A. Shah, Wavelet Transforms and Their Applications, Birkhäuser/Springer, New York, NY, USA, 2nd edition, 2015.

[8] N. Khanna and L. Kathuria, "On convolution of Boas transform of wavelets," Poincare Journal of Analysis and Applications, vol. 6, no. 1, pp. 53-65, 2019. 
[9] N. Khanna, S. K. Kaushik, and A. M. Jarrah, "Some remarks on Boas transforms of wavelets," Integral Transforms and Special Functions, vol. 31, no. 2, pp. 107-117, 2020.

[10] N. Khanna, V. Kumar, and S. K. Kaushik, "Vanishing moments of Hilbert transform of wavelets," Poincare Journal of Analysis and Applications, vol. 2, no. 2, pp. 115-127, 2015.

[11] N. Khanna, V. Kumar, and S. K. Kaushik, "Wavelet packets and their vanishing moments," Poincare Journal of Analysis and Applications, vol. 4, no. 2, pp. 95-105, 2017.

[12] N. Khanna, V. Kumar, and S. K. Kaushik, "Vanishing moments of wavelet packets and wavelets associated with Riesz projectors," in Proceedings of the 12th International Conference on Sampling Theory and Applications (SampTA), pp. 222-226, IEEE, Tallinn, Estonia, July 2017.

[13] S. Mallat, A Wavelet Tour of Signal Processing, Academic Press, San Diego, CA, USA, 1998.

[14] D. F. Walnut, An Introduction to Wavelet Analysis, Applied and Numerical Harmonic Analysis, Birkhäuser Boston, Inc., Boston, MA, USA, 2004.

[15] R. P. Boas, "Some theorems on Fourier transforms and conjugate trigonometric integrals," Transactions of the American Mathematical Society, vol. 40, no. 2, p. 287, 1936.

[16] R. R. Goldberg, "An integral transform related to the Hilbert transform," Journal of the London Mathematical Society, vol. 35, pp. 200-204, 1960.

[17] P. Heywood, "On a transform discussed by Goldberg," Journal of the London Mathematical Society, vol. s1-38, no. 1, pp. 162-168, 1963.

[18] N. H. Zaidi, "On a transform discussed by Goldberg," Journal of the London Mathematical Society, vol. 14, no. 2, pp. 240244, 1976.

[19] A. I. Zayed, Handbook of Function and Generalized Function Transformations, CRC Press, Boca Raton, FL, USA, 1996.

[20] N. Khanna, A. Zothansanga, S. K. Kaushik, and D. Kumar, "Some properties of fractional Boas transforms of wavelets," Journal of Mathematics, vol. 2021, 2021.

[21] L. R. Soares, H. M. de Oliveira, and R. J. Cintra, “The FourierLike and Hartley-Like wavelet analysis based on Hilbert transforms," 2015, https://arxiv.org/abs/1502.02049.

[22] I. Daubechies, "Orthonormal bases of compactly supported wavelets," Communications on Pure and Applied Mathematics, vol. 41, no. 7, pp. 909-996, 1988.

[23] R. S. Pathak, "Variation-diminishing wavelets and wavelet transforms," Real Anal. Exchange, vol. 37, no. 1, pp. 147-166, 2012.

[24] I. J. Schoenberg, "On variation-diminishing integral operators of the convolution type," Proceedings of the National Academy of Sciences, vol. 34, no. 4, pp. 164-169, 1948. 